\title{
Immunological Selection of Variant Mouse Lymphoid Cells with Altered Glucocorticoid Responsiveness
}

\author{
MARK DANIELSEN, ${ }^{1}$ DAVID O. PETERSON,$^{2}$ AND MICHAEL R. STALLCUP ${ }^{1 *}$ \\ Department of Biology, University of South Carolina, Columbia, South Carolina 29208, ${ }^{1}$ and Department of \\ Biochemistry and Biophysics and the Texas Agricultural Experiment Station, Texas A \& M University, \\ College Station, Texas $77843^{2}$
}

Received 2 February 1983/Accepted 25 April 1983

\begin{abstract}
We have devised an immunological procedure to separate cells on the basis of expression of mouse mammary tumor virus (MMTV) gene products. Plastic petri dishes coated with specific antibodies against MMTV proteins bind cells with an efficiency that correlates with the level of MMTV gene expression. Glucocorticoid-sensitive mouse thymoma cell line W7 was infected with MMTV. Clones from the infected population retain the relatively slow cytolytic glucocorticoid response and, in addition, exhibit a rapid induction of MMTV-specific RNA and proteins. By combining our immunological selection with the selection for resistance to hormone-mediated cytolysis, we have isolated variant cells which are resistant to the cytotoxic effect of glucocorticoids but which retain the induction of viral gene products and must therefore have a functional glucocorticoid receptor protein.
\end{abstract}

Like the immunocytes from which they were derived (4), some mouse thymoma cell lines are killed by exposure to physiological concentrations of glucocorticoid hormones for 1 to 3 days $(9,10)$. Although the mechanism of the killing response is not well defined, the hormone-induced cell death provides a strong selection for hormone-insensitive variants. However, the complexity of the killing response and the absence of an identifiable, induced gene product make the biochemical characterization of variant phenotypes difficult. Selection for hormone resistance has yielded several phenotypic classes of variants in the glucocorticoid receptor protein $(11,14,29)$. These have been extremely useful, but other classes of variants would be of obvious interest.

To facilitate the genetic analysis of glucocorticoid responsiveness in lymphoid cells, we have infected them with mouse mammary tumor virus (MMTV), which establishes itself as a stably integrated provirus with glucocorticoid-responsive gene expression $(17,26)$. The infected cells exhibited two independent glucocorticoid responses: the relatively slow cytolytic response and the rapid stimulation of MMTV RNA and protein synthesis. To exploit this phenotype for genetic analysis, we have developed an immunological selection to separate cells on the basis of differential expression of viral proteins on the cell surface. In this report, we describe the characteristics of the MMTV-infected lymphoid cells, the details of our immunological selection procedure, and the selection of variant cells with a novel phenotype: they have lost the cytolytic response to glucocorticoids but retain the ability to induce MMTV gene products and must therefore have a functional glucocorticoid receptor protein.

\section{MATERIALS AND METHODS}

Materials. Dulbecco modified essential medium and horse serum were purchased from Flow Laboratories, Inc. Serum was heat inactivated by incubating $500-\mathrm{ml}$ bottles for $30 \mathrm{~min}$ at $56^{\circ} \mathrm{C}$ and then was clarified by centrifugation. Noble agar was obtained from Difco Laboratories; polystyrene bacterial petri dishes were from Falcon Plastics; ethyl methane sulfonate and dexamethasone (DEX) were from Sigma Chemical Co.; $\left[5,6-{ }^{3} \mathrm{H}\right]$ uridine was from ICN Pharmaceuticals, Inc.; and $\left[\alpha^{32}\right.$ P]dTTP was from New England Nuclear Corp. DNA clone pMTV1 (25) contains the entire MMTV genome (except for a small deleted portion), inserted into the EcoRI site of pBR322.

Cells and tissue culture. All cultures were grown in Dulbecco modified essential medium supplemented with $10 \%$ heat-inactivated horse serum and without antibiotics. W7.2 is a randomly chosen subclone of the BALB/c mouse thymoma-derived cell line WEHI7 (10). GR-A3 is a cloned cell line derived from a mammary tumor in the GR strain of mouse (16). W7 cells were cloned on top of $0.5 \%$ Noble agar in Dulbecco modified essential medium containing $15 \%$ horse serum (14).

For growth curves, cells were counted with a hemacytometer and an inverted phase-contrast microscope. Live cells are large, turgid, translucent spheres that are easily distinguished from dead cells, which are 
shrunken, irregular in shape, and refractile. Viable cell counts obtained by using these visual criteria were identical to those obtained by observing trypan blue exclusion.

Cells were mutagenized by incubation for $16 \mathrm{~h}$ at $37^{\circ} \mathrm{C}$ in growth medium containing $250 \mu \mathrm{g}$ of ethyl methane sulfonate per $\mathrm{ml}$. The surviving cells $(15 \%)$ were grown for 3 days before use in the selection experiments.

Infection of $\mathbf{W} 7$ cells with MMTV. The $\mathrm{C} 3 \mathrm{H}$ strain of MMTV in purified, concentrated form was kindly provided by the Viral Resources Laboratory of the Frederick Cancer Research Center, Frederick, Md. GR strain MMTV preparations for infection of cell cultures were obtained from the 24-h culture supernatant of confluent GR-A3 cultures grown in roller bottles. The frozen supernatant was thawed, centrifuged at $500 \times g$ for $10 \mathrm{~min}$ to remove cell debris, and then centrifuged at $50,000 \times g$ for $3 \mathrm{~h}$ at $4^{\circ} \mathrm{C}$. The viral pellet from $250 \mathrm{ml}$ of supernatant was suspended in 5 ml of Dulbecco modified essential medium with $10 \%$ horse serum and stored frozen at $-70^{\circ} \mathrm{C}$ until use. Cells were infected with MMTV (17) by adding $1 \mathrm{ml}$ of viral concentrate to $1 \mathrm{ml}$ of growth medium containing $10^{7}$ cells. After $2 \mathrm{~h}$ at $37^{\circ} \mathrm{C}$, the cell suspension was diluted 10-fold with fresh growth medium, and after $3 \mathrm{~h}$ more, the cells were washed once with growth medium and suspended in fresh medium.

Anti-MMTV antibodies. Mouse hybridoma line 8G2, used in these studies, secretes monoclonal antibodies against the MMTV protein p27 (D. Ucker, G. Firestone, and $\mathrm{K}$. Yamamoto, personal communication). Monoclonal antibody preparations were obtained by growing the $8 \mathrm{G} 2$ cells as an ascites tumor in BALB/c mice. Ascites fluid was harvested aseptically through a syringe needle, clarified by centrifugation $(10 \mathrm{~min}$ at $2,000 \times g$ ), and stored at $-70^{\circ} \mathrm{C}$. It was used for the immunological selection procedure without further purification.

Preparation of antibody-coated dishes and selection of cells. The procedure for cell selection was based on the "panning" technique of Wysocki and Sato (28). All steps were performed at $4^{\circ} \mathrm{C}$ or on ice; handling of cells and solutions was performed aseptically in a Baker Sterilegard biological safety cabinet. Monoclonal antibody preparations against MMTV protein p27 were diluted with $20 \mathrm{mM}$ Tris-hydrochloride ( $\mathrm{pH} 9.5$ ), and 2 $\mathrm{ml}$ of the solution was loaded into each 60 - $\mathrm{mm}$ bacterial petri dish. The actual dilution (usually ca. 130-fold) was determined empirically for each ascites fluid preparation. Dishes were incubated overnight at $4^{\circ} \mathrm{C}$ and, if not used immediately, were stored with the diluted antibody solution at $-20^{\circ} \mathrm{C}$; dishes retained their cell binding activity for at least a month. Immediately before use, the antibody-coated dishes were washed three times with $5 \mathrm{ml}$ of cold PBSS (phosphatebuffered saline containing 5\% heat-inactivated horse serum), the buffer being removed by aspiration each time. Cells were prepared for selection by being washed once by centrifugation with $10 \mathrm{ml}$ of cold PBSS, resuspended in $2 \mathrm{ml}$ of the same buffer, and then incubated in an antibody-coated dish at $4^{\circ} \mathrm{C}$ for 90 min. The number of cells added to each dish depended on the type of cell being selected. For quantitative binding studies, fewer than $10^{7}$ cells were added to each $60-\mathrm{mm}$ dish. When low cell binding percentages were expected in a selection procedure, as many as $10^{8}$ cells were added to each 100 -mm dish. At 30 and 60 min after cells were added, the dishes were swirled gently to redistribute unbound cells. Unbound cells were removed at $90 \mathrm{~min}$ by careful aspiration, and the dishes were washed gently two times with cold PBSS. Bound cells were removed by repeatedly squirting 2 $\mathrm{ml}$ of cold PBSS onto the bound cells from a Pasteur pipette until complete resuspension of all bound cells was achieved. Bound cells were then washed once with PBSS and resuspended in growth medium. Only viable cells bind to the antibody-coated dishes; dead cells and debris do not bind.

The percentage of cells from a clonal population that bind to antibody-coated dishes is influenced by the amount of antibody loaded onto the dishes. In addition, some lots of horse serum interfere with the binding of cells to the dishes, even though the cell growth rate, DEX-induced cytolytic response, and the DEX induction of MMTV RNA synthesis are normal. Unless stated otherwise below, the serum lot and antibody concentration used for all selections described in this report produced approximately $90 \%$ binding of DEX-treated W7MG1 cells and less than $1 \%$ binding of control W7MG1 cells.

Selection for DEX resistance. Cell populations were alternately grown in medium containing $1 \mu \mathrm{M} D E X$ for $36 \mathrm{~h}$ and DEX-free medium for several days. Some MMTV-infected cells (lymphoma lines and other cell types) grown in DEX for extended periods accumulate very large numbers of integrated MMTV proviral sequences by a mechanism that is not clearly understood but appears not to involve production of infectious virions. Such cells often have very high basal levels of MMTV expression (B. Maler, J. Ring, D. Peterson, and K. Yamamoto, unpublished data). When relatively brief periods of DEX treatment $(36 \mathrm{~h})$ were alternated with periods of DEX-free growth, as in our protocol, the incidence of cells with elevated basal levels of MMTV expression was reduced but not completely eliminated (data not shown).

Measurement of relative intracellular concentrations and rates of synthesis of MMTV RNA. Total cellular MMTV RNA content was determined by two different methods. In Fig. 1 and 2, whole cell RNA from control or 12-h DEX-treated cells was prepared and analyzed for relative content of MMTV RNA by $C_{r} t$ analysis as described by Ringold et al. (19), using MMTV $\left[{ }^{3} \mathrm{H}\right] \mathrm{cDNA}$ prepared as described previously (18). In Fig. 3, a rapid, semiquantitative method for measuring MMTV RNA content of whole cells (D. Peterson and K. Yamamoto, manuscript in preparation) was used. A measured number of cells in phosphate-buffered saline were filtered through a $3-\mathrm{mm}$ chimney onto Whatman 3MM paper. Cells were lysed in situ by freezing and thawing, fixed with ethanol-acetic acid (3:1), washed with $95 \%$ ethanol, and dried at room temperature. The filter was hybridized to pMTV1 $\left.{ }^{32} \mathrm{P}\right] \mathrm{DNA}$ prepared by nick translation (15), washed, and subjected to autoradiography (21). The dried filter was then stained with $0.2 \%$ Coomassie blue in methanol-acetic acid (7:1) and destained in 95\% ethanol. The stained filter gives an indication of the relative number of cells fixed to each spot, and the autoradiogram indicates the relative amount of MMTV RNA on each spot.

The relative rate of synthesis of MMTV RNA was determined by nucleic acid filter hybridization analysis 

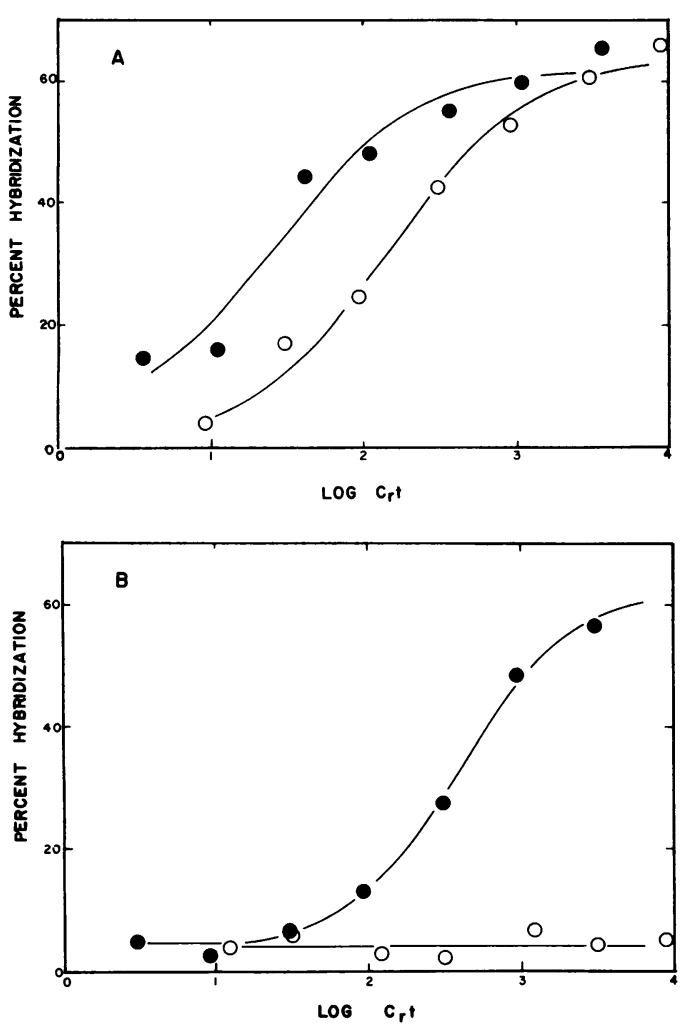

FIG. 1. Concentration of MMTV RNA in clones of infected W7.2. The relative concentration of RNA was determined by $\mathrm{C}_{\mathrm{r}} \mathrm{t}$ analysis as described in the text. Panel A, W7MG1 cells; Panel B, W7M4.12 cells. Symbols: $\bigcirc$, no DEX treatment; 9 , cells treated for 12 $h$ with $1 \mu M$ DEX.

of RNA from pulse-labeled cells. A total of $10^{7}$ cells were grown in $5 \mathrm{ml}$ of normal growth medium for $\mathbf{3 0}$ $\min$ in the presence or absence of $1 \mu \mathrm{M}$ DEX; 0.25 $\mathrm{mCi}$ of $\left[{ }^{3} \mathrm{H}\right]$ uridine was added for $15 \mathrm{~min}$, and then RNA was isolated from the cells (19). The relative amount of MMTV RNA in the $\left[{ }^{3} \mathrm{H}\right] \mathrm{RNA}$ preparations was determined by hybridization to nitrocellulose filters containing immobilized pMTV1 DNA, as described by McKnight and Palmiter (13) and modified by Ucker et al. (25).

\section{RESULTS}

Characterization of MMTV-infected W7 cells. W7 cells are killed by a 36- to 72-h exposure to glucocorticoid concentrations as low as $5 \mathrm{nM}$; the response is mediated by the glucocorticoid receptor protein (1). Like all normal BALB/c cells, W7 cells carry two complete copies of MMTV proviral DNA as well as a third, incomplete copy (5). These endogenous viral genes are not expressed in W7 or in any normal BALB/c mouse tissue examined $(23,27)$.

W7.2 was infected with the $\mathrm{C} 3 \mathrm{H}$ strain of MMTV. In contrast to uninfected W7.2 cells,
MMTV RNA was readily detected in the infected population after treatment with $1 \mu \mathrm{M}$ DEX for $12 \mathrm{~h}$. Cell viability was not affected by exposure to $1 \mu \mathrm{M}$ DEX for as much as $14 \mathrm{~h}$, if cells were promptly returned to DEX-free medium.

Two clones of infected cells (W7MG1 and W7M4.12) were characterized for their contents of MMTV RNA and DNA. MMTV RNA was quantitated by solution hybridization. W7MG1 (Fig. 1A) had a significant level of MMTV RNA in the absence of DEX, and after a 12-h hormone treatment, the steady-state level of viral RNA increased about 10-fold to a level corresponding to about $0.07 \%$ of total cellular RNA (based on $\mathrm{C}_{\mathrm{r}} \mathrm{t}_{1 / 2}$ of 0.02 for pure MMTV RNA). W7M4.12 (Fig. 1B), on the other hand, had no detectable MMTV RNA in the absence of hormone, and DEX caused at least a 100-fold induction of viral

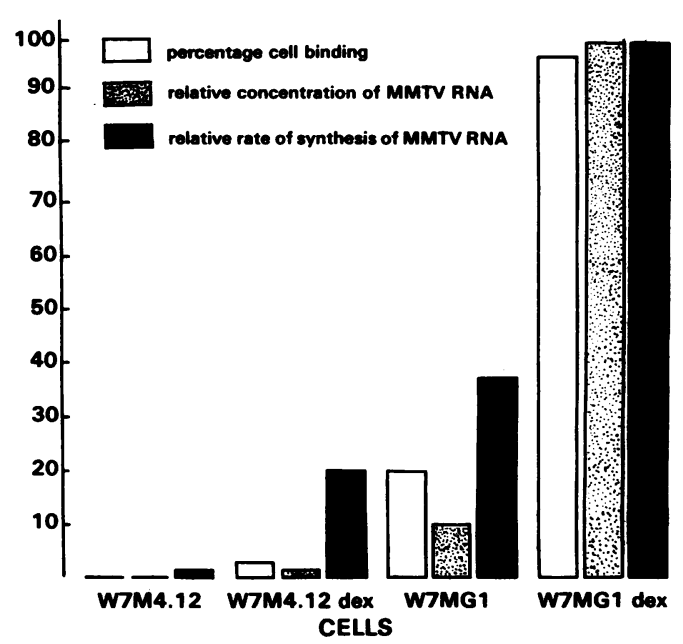

FIG. 2. Comparison of cell binding to antibodycoated dishes and MMTV RNA synthesis. The percentage of total cells that bind to antibody-coated dishes was determined as described in the text, using control cells or cells grown with $1 \mu M$ DEX for $8 \mathrm{~h}$. Each bar on the graph represents the average of five or more independent determinations. Variation from the mean was 5 percentage points or less. Full scale (100 units) on the graph represents $100 \%$ binding of cells. The relative intracellular concentration of MMTV RNA was determined by $\mathrm{C}_{\mathrm{r}} \mathrm{t}$ analysis as described in the text, using RNA from control cells or cells grown with $1 \mu \mathrm{M}$ DEX for $12 \mathrm{~h}$. Full scale (100 units) on the graph represents a relative MMTV RNA concentration of $0.07 \%$ of total cell RNA. Each bar represents the results of one $\mathrm{C}_{\mathrm{r}} \mathrm{t}$ analysis. The relative rate of MMTV RNA synthesis was determined by filter h: bridization analysis of pulse-labeled cell RNA, as described in the text. Full scale (100 units) on the graph represents a relative rate of MMTV RNA synthesis of $0.45 \%$ of total $\left[{ }^{3} \mathrm{H}\right] \mathrm{RNA}$. Each bar represents the average of two determinations; for each value, variation from the mean was 5 percentage points or less. 


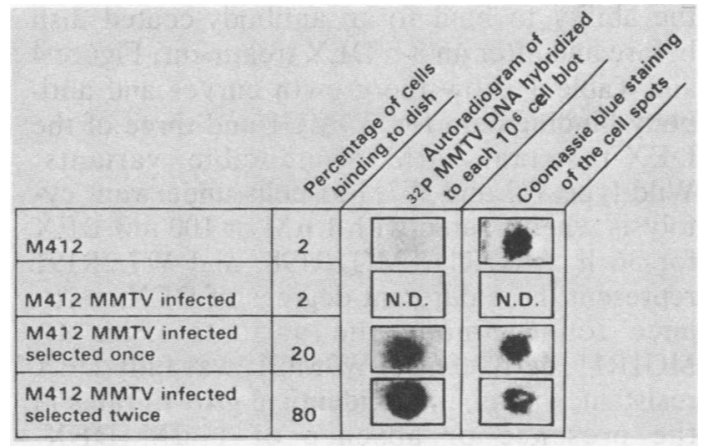

FIG. 3. Selection of cells with high levels of MMTV expression from an infected population. W7M4.12 cells were reinfected with MMTV and subjected to two rounds of petri dish selection for MMTV expression in the presence of DEX, as described in the text. At each step of the procedure, a sample of cells was treated with DEX for $8 \mathrm{~h}$ and analyzed for binding to an antibody-coated petri dish. The percentage of total cells that bound to the dish is shown in the lefthand column. A sample of $10^{6} \mathrm{DEX}$-treated cells was also analyzed for MMTV RNA content by the whole cell filter hybridization technique (see the text). The middle column shows the autoradiogram of the cell dots after hybridization with MMTV $\left[{ }^{32}\right.$ P $] D N A$. The right-hand column shows the same cell dots stained with Coomassie blue.

RNA to a final concentration of about $0.005 \%$ of total cellular RNA.

A Southern blot analysis of cellular DNA (22) was carried out with $E c o$ RI, which cleaves the BALB/c endogenous MMTV provirus (5) and the $\mathrm{C} 3 \mathrm{H}$ exogenously acquired provirus (21) once. In addition to the endogenous MMTV genes, W7MG1 had 10 copies of proviral DNA, and W7M4.12 had 8 copies (data not shown).

Correlation of MMTV RNA synthesis with cell binding to antibody-coated dishes. Our preliminary observations suggested that some MMTVinfected W7 cells accumulate virus-specific cell surface proteins in response to DEX. To exploit this observation, we adapted a procedure that employs antibody-coated petri dishes (see above) to separate cells that are expressing viral cell surface proteins from cells that are not. To test the specificity of this procedure, W7.2, W7M4.12, and W7MG1 cells, grown in the presence or absence of DEX, were analyzed by three parameters: (i) binding to petri dishes coated with MMTV-specific antibodies, (ii) intracellular concentrations of MMTV RNA, and (iii) rate of synthesis of MMTV RNA. Uninfected W7.2 cells (control or DEX-treated) contained no detectable MMTV RNA and exhibited no measurable binding to an antibody-coated dish. However, W7M4.12 and W7MG1 cells bound to the dishes, and the amount of binding correlated with the concentration and rate of synthesis of MMTV RNA in the cell (Fig. 2). In the absence of DEX, W7M4.12 cells had a very low level of MMTV RNA synthesis and did not bind to an antibody-coated dish, but after DEX treatment, we saw a 30-fold stimulation in the rate of MMTV RNA synthesis, and $1 \%$ of the cells bound (background binding was about $0.1 \%$ ). For W7MG1, 20\% of the cells bound before DEX treatment, and $97 \%$ bound after $8 \mathrm{~h}$ in DEX. In all cases, the percentage of cells that bound correlated with the level and rate of synthesis of MMTV RNA in the cell.

Selection of cells with high levels of MMTV gene expression. To test the feasibility of using our immunological method to isolate cells with a rare phenotype from a large population, we reinfected a culture of $10^{7}$ W7M4.12 cells with MMTV virions. Such an infected population was expected to contain cells with different levels of MMTV gene expression when treated with DEX; we applied our immunological selection to isolate a subpopulation containing relatively high levels of MMTV gene products. The reinfected W7M4.12 cells were grown for 3 days and treated with DEX for $8 \mathrm{~h}$, and then $6 \times 10^{6}$ cells were incubated in an antibody-coated dish. The bound cells (fewer than $10^{5}$ cells) were removed from the dish, grown for 6 days in the absence of DEX, and treated for $8 \mathrm{~h}$ with DEX, and $6 \times 10^{6}$ cells were subjected to a second round of the immunological selection. Again, antibodybound cells were removed and grown without DEX. At each step of the selection scheme, a sample of cells was treated with DEX for $8 \mathrm{~h}$ and used to determine the percentage of cells that would bind to an antibody-coated dish; the intracellular MMTV RNA concentration of each DEX-treated population was also measured by the whole cell filter hybridization procedure described above.

Before the reinfection, W7M4.12 cells bound very poorly (2\%) to an antibody-coated dish and contained low levels of MMTV RNA when induced with DEX (Fig. 3). After reinfection, there was no change in the binding of the DEXtreated cells to an antibody-coated dish. Apparently, only a very small fraction of the reinfected cells made enough MMTV proteins to bind strongly to the dish. Each round of immunological selection enriched the population for cells expressing high levels of MMTV RNA and MMTV cell surface proteins (Fig. 3). Thus, the selection procedure appears to be suitable for separating a small number of cells with high MMTV expression from a large population of cells with generally low levels of MMTV gene expression.

Selection of DEX-resistant, MMTV-inducible variants. W7MG1 was chosen as the parent cell 
line for selection of variants resistant to the cytolytic effect of glucocorticoids but retaining the hormone-mediated stimulation of MMTV gene expression. W7MG1 cells bound well (90\%) to MMTV antibody-coated petri dishes after DEX treatment, but did not bind $(\sim 0.1 \%)$ without DEX treatment. An ethyl methane sulfonate-treated population $\left(10^{8}\right.$ cells) was grown with DEX for $8 \mathrm{~h}$ and then was distributed among four 100-mm antibody-coated dishes; the unbound cells were discarded. To select for DEX resistance, the bound cells were removed from the dish and incubated in medium with DEX for an additional $28 \mathrm{~h}$ before being placed in medium without DEX. To remove cells with elevated basal levels of MMTV expression (see above), the selected populations were grown for at least $\mathbf{1 0}$ generations in the absence of DEX and then were exposed to antibody-coated dishes $\left(10^{7}\right.$ cells on three dishes). This time, bound cells were discarded, and the unbound cells were treated with DEX for $\mathbf{8} h$ and again selected for MMTV expression. At this point, after one complete cycle of the selection protocol, less than $5 \%$ of the DEX-resistant population bound to the antibody-coated dish after DEX treatment. After four cycles of selection, the resulting population appeared to be composed mostly of cells that were DEX resistant and inducible for MMTV expression. Clones of this population were obtained as described above for analysis of phenotype.

A second approach was used to obtain DEXresistant, MMTV-inducible variants from the uninfected W7.2 clone. An ethyl methane sulfonate-mutagenized population of $10^{8} \mathrm{~W} 7.2$ cells was repeatedly alternated between growth medium containing $1 \mu \mathrm{M}$ DEX for $36 \mathrm{~h}$ and growth medium without DEX for several days; after four such cycles, a DEX-resistant population was obtained. Based upon previous studies (11, $14,29)$, we expected the majority of these cells to be receptor variants. Furthermore, we assumed that some cells with lesions affecting components other than the receptor protein should still be able to carry out other glucocorticoid responses. To identify potential variants of this type, we infected the DEX-resistant population with GR strain MMTV, treated the cells with DEX for $8 \mathrm{~h}$, and then used antibodycoated dishes to select for cells with high levels of MMTV expression. The bound cells were removed, propagated, and reselected for DEXinducible MMTV expression. After three rounds of selection, random clones were obtained from the selected population for analysis.

Phenotype analysis of variant clones. Random clones from the two selected populations described above were characterized for growth rate in the presence and absence of DEX and for the ability to bind to an antibody-coated dish before and after an 8-h DEX treatment. Figure 4 and Table 1 show the growth curves and antibody-binding data for W7MG1 and three of the DEX-resistant, MMTV-inducible variants. Wild-type W7 and W7MG1 cells underwent cytolysis when treated with $1 \mu \mathrm{M}$ or $100 \mathrm{nM}$ DEX for $36 \mathrm{~h}$. MG1R11, MG1R198, and W7.2R191 represent three different degrees of DEX resistance found among the variants (Fig. 4). MG1R11, derived from W7MG1, was fully DEX resistant, that is, it had identical growth rates in the presence or absence of $1 \mu M$ DEX. MG1R198, also derived from W7MG1, was killed by $1 \mu \mathrm{M}$ DEX (although more slowly than the parent W7MG1), but it grew at a reduced rate in $100 \mathrm{nM}$ DEX. W7.2R191, derived from W7.2, was growth inhibited by $1 \mu \mathrm{M}$ DEX but remained viable for over a week before undergoing cytolysis. Quantitative measurements of cell binding to antibody-coated dishes (Table 1) showed that all three DEX-resistant variants in Fig. 4 had DEX-inducible MMTV expression. The elevated basal level of MMTV expression in MG1R11 may indicate that this clone has acquired additional copies of the viral genome, as discussed above. MG1R198 had a reduced level of DEX-induced MMTV expression; because this clone was only partially resistant to DEX, it may have reduced levels of glucocorticoid receptors. Characterization of the glucocorticoid receptor levels in these and other variant clones

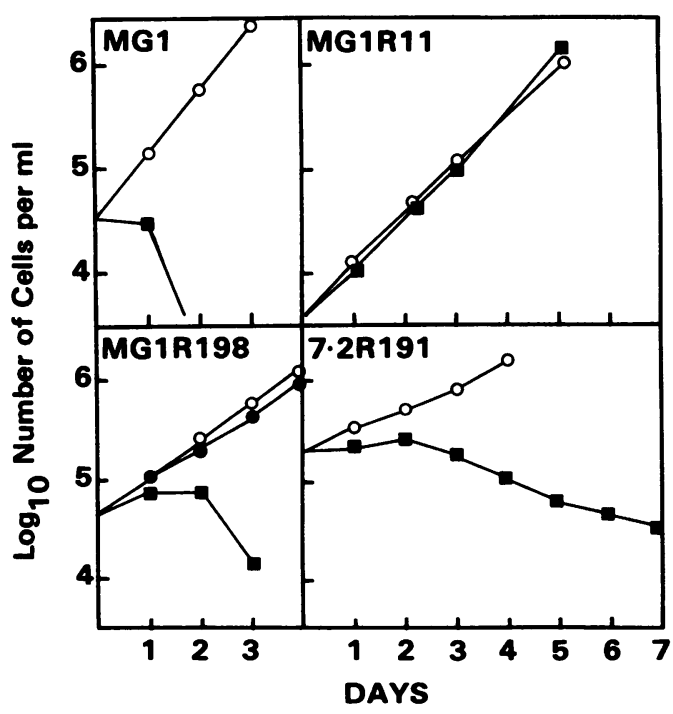

FIG. 4. Growth curves of parental cells and three DEX-resistant, MMTV-inducible variants. Each clone was grown in the absence or presence of DEX. Only viable cells were counted, as described in the text. Symbols: O, no DEX; O, 100 nM DEX; $\square, 1 \mu M$ DEX. 
TABLE 1. Binding of parental and DEX-resistant clones to antibody-coated dishes

\begin{tabular}{lcc}
\hline \multirow{2}{*}{ Clone } & \multicolumn{2}{c}{$\%$ Bound cells $^{a}$} \\
\cline { 2 - 3 } & -DEX & +DEX \\
\hline W7MG1 & $<1$ & 87 \\
MG1R11 & 24 & 62 \\
MG1R198 & $<1$ & 13 \\
W7.2R191 & 2 & 85 \\
\hline
\end{tabular}

${ }^{a}$ Cells of each clone were grown without DEX $(-D E X)$ or with $1 \mu M$ DEX (+DEX) for $8 \mathrm{~h}$; the percentages of control and DEX-treated cells that bound to antibody-coated dishes were determined as described in the text.

with the same phenotype is currently in progress. DEX inducibility of MMTV RNA synthesis in some DEX-resistant clones was verified by pulse-labeling whole cells with $\left[{ }^{3} \mathrm{H}\right]$ uridine and measuring MMTV $\left[{ }^{3} \mathrm{H}\right] \mathrm{RNA}$ by filter hybridization (data not shown).

Table 2 shows a summary of the phenotypes of all of the clones examined from the final selected populations. Approximately one-third of the clones selected from the W7MG1 parent line had the desired phenotype: MMTV inducible and either fully or partially DEX resistant. The fraction was lower for the W7.2 selection, but several clones of the desired phenotype were obtained. The remainder of the clones examined either had the parental phenotype (MMTV inducible, DEX sensitive) or had lost both responses (MMTV uninducible, DEX resistant).

\section{DISCUSSION}

We have developed an immunological proceI dure for fractionating cultured mouse lymphoma cells according to their ability to bind to petri dishes coated with a monoclonal antibody against MMTV cell surface proteins. The procedure is technically simple to perform and requires no sophisticated instrumentation. Very large populations of cells can be processed rapidly (in about $2 \mathrm{~h}$ ). As many as $5 \times 10^{7}$ cells can be bound to a $100-\mathrm{mm}$ dish. When a population contains a low percentage of cells that will bind to the dishes, $10^{8}$ cells can be loaded into each $100-\mathrm{mm}$ dish without reducing the efficiency of binding of antigen-positive cells. Relatively high levels of expression of MMTV functions are required for efficient binding to the antibodycoated dish.

The monoclonal antibody preparation we have used is specific for MMTV protein p27 (D. Ucker, G. Firestone, and K. Yamamoto, personal communication). In the MMTV virion, p27 is part of the nucleoprotein core and is not accessible to antibodies (20) or to iodination (24) unless the virion is disrupted with detergents.
Callis and Ritzi (3) reported that p27 in whole, cultured mouse mammary tumor cells is not accessible to antibodies. In contrast, our results indicate that p27 in whole, MMTV-infected W7 cells can interact with antibodies. The analogous capsid protein from another retrovirus, murine leukemia virus, has also been observed on the outer surface of infected lymphoid cells (2).

Using a fluorescence-activated cell sorter and antibodies against MMTV envelope glycoprotein gp52, Grove et al. (7) have devised an immunological procedure for obtaining glucocorticoid response variants of MMTV-infected HTC cells. As was the case in our studies, they succeeded in isolating variants that had lost some glucocorticoid responses but retained others (8). The cell sorter can effectively discriminate lower levels of antigen than can our petri dish method; however, the processing of $10^{8}$ or more cells, which is easily and quickly accomplished with petri dishes, would require many hours and considerable expense with a cell sorter.

By choosing the nonbinding cell fraction from a DEX-treated population, our immunological technique can be used to select cells that have lost MMTV inducibility. The efficiency of selection for antigen-negative cells is lower due to incomplete binding of some antigen-positive cells; nevertheless, repeated rounds of selection have eventually yielded noninducible cells from a mutagenized population of W7MG1 cells (unpublished data). The selection for nonbinding cells provides an alternative to the antibodymediated complement lysis technique for obtain-

TABLE 2. Summary of clone phenotypes from populations enriched for DEX-resistant, MMTVinducible cells ${ }^{a}$

\begin{tabular}{|c|c|c|c|c|c|}
\hline \multirow{2}{*}{$\begin{array}{l}\text { Parent } \\
\text { cell line }\end{array}$} & \multirow{2}{*}{$\begin{array}{l}\text { Total no. } \\
\text { of clones } \\
\text { examined }\end{array}$} & \multicolumn{4}{|c|}{ No. of clones of the following type: } \\
\hline & & $\begin{array}{c}\text { MMTV }^{\mathbf{1}} \\
\text { DEX }^{\mathbf{2}}\end{array}$ & $\begin{array}{l}\text { MMTVi }^{\mathbf{1}} \\
\text { DEX }^{\mathrm{pr}}\end{array}$ & $\begin{array}{c}\text { MMTV }^{\mathbf{1}} \\
\text { DEX }^{s}\end{array}$ & $\begin{array}{c}\text { MMTV }^{u} \\
\text { DEX }^{r}\end{array}$ \\
\hline W7MG1 & 164 & 24 & 27 & 22 & 91 \\
\hline W7.2 & 26 & 1 & 2 & 10 & 13 \\
\hline
\end{tabular}

a Random clones were obtained from the final selected populations as described in the text. Each clone was assayed for DEX resistance by growth curve analysis with various DEX concentrations and for MMTV inducibility by binding of control and $1 \mu \mathrm{M}$ DEX-treated cells to antibody-coated dishes. The number of clones representing each phenotype is listed in the table. Abbreviations: MMTVi, DEX-inducible MMTV expression; MMTVu, MMTV expression uninducible by DEX; DEX ${ }^{r}$, DEX resistant: no growth inhibition by $1 \mu \mathrm{M}$ DEX; DEX ${ }^{\mathrm{pr}}$, partially resistant to DEX: growth inhibition or cytolysis with $1 \mu \mathrm{M} \mathrm{DEX}$, but little or no growth inhibition by $100 \mathrm{nM}$ DEX; DEX , DEX sensitive: killed within 2 to 3 days by 100 nM DEX. 
ing antigen-negative variants $(6,12)$. The antibody-coated petri dish method may prove useful as a positive or negative selection for genetic studies involving other antigens found in relatively large numbers on the cell surface.

The immunological procedure described in this report was used to isolate rare MMTVinducible cells from a large DEX-resistant population. Because they retain the glucocorticoid receptor-mediated induction of MMTV gene products, these cells must possess functional glucocorticoid receptor proteins. They therefore define a novel class of glucocorticoid-resistant lymphoid cells.

\section{ACKNOWLEDGMENTS}

This project was initiated in the laboratory of Keith $\mathbf{R}$. Yamamoto. We are grateful to him for many helpful discussions and for critical reading of the manuscript. We also thank Debra Chavis and Sue Timmerman for expert preparation of the manuscript.

This work was supported by grants from the American Cancer Society (no. IN107E to M.R.S.), the National Institutes of Health (no. GM28298 to M.R.S. and no. CA32695 to D.O.P.), and the Helen Hay Whitney Foundation (to D.O.P.). This research was also partially funded by the Texas Agricultural Experiment Station (to D.O.P.).

\section{LITERATURE CITED}

1. Bourgeois, S., and R. F. Newby. 1977. Diploid and haploid states of the glucocorticoid receptor gene of mouse lymphoid cell lines. Cell 11:423-430.

2. Buetti, E., and H. Diggelmann. 1980. Murine leukemia virus proteins expressed on the surface of infected cells in culture. J. Virol. 33:936-944.

3. Callis, A. H., and E. M. Ritzi. 1980. Detection and characterization of mouse mammary tumor virus cell surface antigens: estimation of antigen abundance by protein A assay. J. Virol. 35:876-887.

4. Claman, H. N. 1972. Corticosteroids and lymphoid cells. N. Engl. J. Med. 24:388-397.

5. Cohen, J. C., J. E. Majors, and H. E. Varmus. 1979. Organization of mouse mammary tumor virus-specific DNA endogenous to BALB/c mice. J. Virol. 32:483-496.

6. Firestone, G. L., and K. R. Yamamoto. 1983. Two classes of mutant mammary tumor virus-infected HTC cell with defects in glucocorticoid-regulated gene expression. Mol. Cell. Biol. 3:149-160.

7. Grove, J. R., B. S. Dieckmann, T. A. Schroer, and G. M. Ringold. 1980. Isolation of glucocorticoid-unresponsive rat hepatoma cells by fluorescence-activated cell sorting. Cell 21:47-56.

8. Grove, J. R., and G. M. Ringold. 1981. Selection of rat hepatoma cells defective in hormone regulated production of mouse mammary tumor virus RNA. Proc. Natl. Acad. Sci. U.S.A. 78:4349-4353.

9. Harris, A. W. 1970. Differentiated functions expressed by cultured mouse lymphoma cells. Exp. Cell Res. 60:341353.

10. Harris, A. W., A. D. Bankhurst, S. Mason, and N. L. Warner. 1973. Differentiated functions expressed by cultured mouse lymphoma cells, II. 0-Antigen, surface immunoglobin and a receptor for antibody on cells of a thymoma cell line. J. Immunol. 110:431-438.

11. Huet-Minkowski, M., J. C. Gasson, and S. Bourgeois. 1981. Induction of glucocorticoid resistant variants in a murine thymoma line by antitumor drugs. Cancer Res.
41:4540-4546.

12. Kabat, D., M. Ruta, M. J. Murray, and E. Polonoff. 1980. Immunoselection of mutants deficient in cell surface glycoproteins encoded by murine erythroleukemia viruses. Proc. Natl. Acad. Sci. U.S.A. 77:57-61.

13. McKnight, G. S., and R. D. Palmiter. 1979. Transcriptional regulation of the ovalbumin and conalbumin genes by steroid hormones in chick oviduct. J. Biol. Chem. 254:9050-9058.

14. Pfahl, M., R. J. Kelleher, and S. Bourgeois. 1978. General features of steroid resistance in lymphoid cell lines. Mol. Cell. Endocr. 10:193-207.

15. Rigby, P. W. J., M. Dieckman, C. Rhodes, and P. Berg. 1977. Labeling deoxyribonucleic acid to high specific activity in vitro by nick translation with DNA polymerase I. J. Mol. Biol. 113:237-251.

16. Ringold, G., E. Y. Lasfargues, J. M. Bishop, and H. E. Varmus. 1975. Production of mouse mammary tumor virus by cultured cells in the absence and presence of hormones: assay by molecular hybridization. Virology 65:135-147.

17. Ringold, G. M., R. D. Cardiff, H. E. Varmus, and K. R. Yamamoto. 1977. Infection of cultured rat hepatoma cells by mouse mammary tumor virus. Cell 10:11-18.

18. Ringold, G. M., P. R. Shank, H. E. Varmus, J. Ring, and K. R. Yamamoto. 1979. Integration and transcription of mouse mammary tumor virus DNA in rat hepatoma cells. Proc. Natl. Acad. Sci. U.S.A. 76:665-669.

19. Ringold, G. M., K. R. Yamamoto, G. M. Tomkins, J. M. Bishop, and H. E. Varmus. 1975. Dexamethasone-mediated induction of mouse mammary tumor virus RNA: a system for studying glucocorticoid action. Cell 6:299-305.

20. Sarkar, N. H., and A. S. Dion. 1975. Polypeptides of the mouse mammary tumor virus. I. Characterization of two group-specific antigens. Virology 64:471-491.

21. Shank, P. R., J. C. Cohen, H. E. Varmus, K. R. Yamamoto, and G. M. Ringold. 1978. Mapping of linear and circular forms of mouse mammary tumor virus DNA with restriction endonucleases: evidence for a large specific deletion occurring at high frequency during circularization. Proc. Natl. Acad. Sci. U.S.A. 75:2112-2116.

22. Southern, E. M. 1975. Detection of specific sequences among DNA fragments separated by gel electrophoresis. J. Mol. Biol. 98:503-517.

23. Stallcup, M. R., J. C. Ring, D. S. Ucker, and K. R. Yamamoto. 1979. Mammary tumor virus genes: probes for mechanisms of transcriptional regulation. Cold Spring Harbor Conf. Cell Proliferation 6:919-936.

24. Teramoto, Y. A., M. J. Puentes, L. J. T. Young, and R. D. Cardiff. 1974. Structure of the mouse mammary tumor virus: polypeptides and glycoproteins. J. Virol. 13:411418.

25. Ucker, D. S., S. R. Ross, and K. R. Yamamoto. 1981. Mammary tumor virus DNA contains sequences required for its hormone-regulated transcription. Cell 27:257-266.

26. Vaidya, A. B., E. Y. Lasfargues, G. Heubel, J. C. Lasfargues, and D. H. Moore. 1976. Murine mammary tumor virus: characterization of infection of nonmurine cells. J. Virol. 18:911-917.

27. Varmus, H. E., N. Quintrell, E. Medeiros, J. M. Bishop, R. C. Nowinski, and N. H. Sarkar. 1973. Transcription of mouse mammary tumor virus genes in tissues from high and low tumor incidence mouse strains. J. Mol. Biol. 79:663-679.

28. Wysocki, L. J., and V. L. Sato. 1978. "Panning" for lymphocytes: a method for cell selection. Proc. Natl. Acad. Sci. U.S.A. 75:2844-2848.

29. Yamamoto, K. R., M. R. Stampfer, and G. M. Tomkins. 1974. Receptors from glucocorticoid-sensitive lymphoma cells and two classes of insensitive clones: physical and DNA-binding properties. Proc. Natl. Acad. Sci. U.S.A. 71:3901-3905. 